$>$ La schizophrénie et le trouble bipolaire sont des maladies neuropsychiatriques à l'étiologie méconnue. Ces deux pathologies présentent des mécanismes étiopathogéniques, génétiques, environnementaux et immuno-inflammatoires communs. La réactivation d'un rétrovirus endogène humain de type $W$ (HERV-W) pourrait être à l'origine de cette physiopathologie partagée. Nous présentons les études mettant en évidence HERV-W chez les patients schizophrènes et bipolaires. Nous proposerons ensuite un modèle physiopathologie en deux temps pouvant être à l'origine de la maladie. L'identification de sous-groupes immuno-inflammatoires associés à une réactivation de HERV-W ouvrirait la voie à l'utilisation de biomarqueurs diagnostiques et de thérapeutiques ciblées, premier pas vers le développement d'une psychiatrie personnalisée pour les patients. <

La schizophrénie ( $\mathrm{SCZ}$ ) et le trouble bipolaire (TB) sont des maladies psychiatriques complexes résultant de l'interaction entre des facteurs génétiques et des facteurs environnementaux. La physiopathologie de ces deux maladies est partiellement connue. Elles partagent, en partie, certains facteurs génétiques et environnementaux ainsi que de nombreux symptômes, rendant le diagnostic différentiel difficile dans certains cas [1]. Ce recouvrement partiel de la symptomatologie pose la question de voies communes entre SCZ et le TB. Depuis quelques années, de nombreuses études ont montré le rôle important d'anomalies immunoinflammatoires dans la physiopathologie des deux maladies, comme en témoigne l'importance des comorbidités auto-immunes que présentent les patients [2]. Certains cas de psychoses ont été directement reliés à l'activité d'autoanticorps anti-synapses, ces cas étant réversibles par un traitement immunomodulateur adapté [3]. Ces observations ont conduit à s'intéresser

Vignette (Photo @ Inserm - Alexia Daoust).

\section{Les rétrovirus endogènes humains, une implication dans la schizophrénie et le trouble bipolaire}

Pierre Ellul $^{1,2,4}$, Laurent Groc ${ }^{3,4}$, Marion Leboyer ${ }^{1,2,4}$

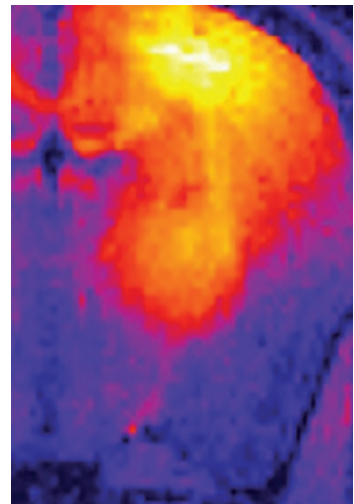

'Pôle psychiatrie des hôpitaux universitaires Henri Mondor, AP-HP, université Paris- $\varepsilon s t$, DHU PePSY, hôpital Albert Chenevier, 40, rue de Mesly, 94000 Créteil, France;

${ }^{2}$ Inserm U955, équipe 15, psychiatrie translationnelle, 94000 Créteil, France ;

${ }^{3}$ Institut interdisciplinaire de neuroscience, CNRS UMR 5297, Université de Bordeaux, 33076 Bordeaux, France ;

plus avant aux mécanismes immunoinflammatoires qui seraient impliqués dans la SCZ et le TB, plus précisément dans certains sous-groupes de pathologies que nous pourrions qualifier ${ }^{4}$ Fondation FondaMental, 94000 Créteil, France. pierre.ellul1987@gmail.com laurent.groc@u-bordeaux.fr marion.leboyer@inserm.fr de «psychoses inflammatoires». Une piste prometteuse reliant facteurs génétiques, environnementaux et immunité est apparue depuis quelques années avec la mise en évidence du rôle possible de la réactivation d'un rétrovirus humain endogène de la famille W (HERV-W) chez ces patients. Dans cette revue, nous ferons une brève description des rétrovirus humains endogènes $[54](\rightarrow)$.

Nous ferons ensuite l'état des lieux des études qui

$\rightarrow$ Voir la Synthèse de J. Medina et H. Perron, $m / s n^{\circ} 2$, février 2017, page 151 établissent un lien entre ces HERV et la SCZ et les TB. Nous terminerons en proposant une hypothèse mécanistique à l'origine du développement de ces maladies en décrivant les nouvelles pistes thérapeutiques qui peuvent être envisagées.

\title{
Les rétrovirus endogènes humains
}

Le décodage du génome humain a permis de montrer que l'ADN de l'homme comprenait $42 \%$ d'éléments répétés, transposables ou rétro-transposables [4]. Les éléments rétro-transposables peuvent être divisés en deux sous-types selon qu'ils présentent ou non des séquences flanquantes répétées dites long terminal repeat (LTR). Les rétro-éléments possédant ces LTR correspondent aux rétrovirus endogènes ( $\varepsilon R V$; quand il s'agit du génome humain, on parle d'HERV pour 


\begin{tabular}{|llll}
\hline Publications & $\begin{array}{l}\text { Observations } \\
\text { faites dans la SCZ } \\
\text { (nombre de patients) }\end{array}$ & $\begin{array}{l}\text { Observations } \\
\text { faites dans le TB } \\
\text { (nombre de patients) }\end{array}$ & Méthodes \\
Yolken et al. (2000) [24] & $\begin{array}{l}\text { Augmentation de l'expression de } \\
\text { HERV-W pol (4) }\end{array}$ & $\begin{array}{l}\text { Augmentation de l'expression de } \\
\text { HERV-K10 pol (4) }\end{array}$ & RT-PCR dans le lobe préfrontal \\
\hline Weis et al. (2007) [25] & $\begin{array}{l}\text { Diminution de l'expression de } \\
\text { HERV-W gag dans le CCA et l'hippo- } \\
\text { campe (15) }\end{array}$ & $\begin{array}{l}\text { Diminution de l'expression de } \\
\text { HERV-W gag dans l'hippocampe } \\
\text { (15) }\end{array}$ & Immunohistochimie, Western blot \\
Frank et al. (2005) [23] & $\begin{array}{l}\text { Augmentation de l'expression de } \\
\text { HERV-K10 pol (35) }\end{array}$ & $\begin{array}{l}\text { Augmentation de l'expression de } \\
\text { HERV-K10 pol (35) }\end{array}$ & Micro-array (puces)
\end{tabular}

Tableau I. Présence des HERV dans les échantillons cérébraux de patients schizophrènes et bipolaires. HERV-W : human endogenous retrovirus type W; SCZ : schizophrénie : TB : trouble bipolaire; RT-PCR : reverse transcriptase-polymerase chain reaction; CCA : cortex cingulaire antérieur.

human endogenous retroviruses) [5]. La découverte de l'existence de ces HERV remonte à la fin des années 1960 [6]. La plupart ont intégré le génome de primates, il y a plusieurs millions d'années, par l'infection de gamètes avec rétro-intégration de copies dites «provirales» dans I'ADN chromosomique avec dissémination intra-génomique et réinfections multiples par la souche environnementale pendant une longue période $[7,8]$. Les propriétés de ces éléments génétiques, aussi qualifiés de «mobiles», ont permis de créer de nouvelles insertions par rétro-transposition dans le génome hôte. Parallèlement, des réinfections multiples, au cours de l'évolution, expliquent aussi l'addition de copies homologues dans le génome des espèces concernées [9]. D'une certaine manière, les HERV peuvent être considérés comme des cicatrices d'infections rétrovirales qui se sont accumulées au cours de l'évolution et qui se sont maintenues jusqu'à l'homme. Ils représentent aujourd'hui $8 \%$ de l'ensemble du génome humain. La majorité des HERV sont retrouvés de manière ubiquitaire dans I'ADN, que leur séquence soit complète ou non [4]. Cependant, certaines copies ne sont présentes dans le génome que d'une partie de la population humaine [10]. Du point de vue nucléotidique, les HERV sont constitués de quatre gènes principaux [11] : (1) gag qui code les protéines de la capside, de la nucléocapside et de la matrice; (2) pro qui code la protéase; (3) pol qui code la transcriptase inverse et l'intégrase (enzymes permettant respectivement, la conversion de l'ARN en ADN et l'intégration au sein de l'ADN de l'hôte) et (4) env qui code la protéine d'enveloppe. L'expression de ces gènes est normalement régulée par l'activité des deux séquences LTR situées en 5' et 3 ' de l'ADN [12]. Parmi les différents HERV, le sous-type $W(H \varepsilon R V-W$ ) semble particulièrement impliqué en psychiatrie. Au moins 140 séquences appartenant à la famille HERV-W ont pu être identifiées dans l'ADN humain à ce jour dont seulement 40 présentent une séquence complète [13]. Le HERV-W a une distribution chromosomique ubiquitaire à l'exception, actuellement connue, des chromosomes 16 et 22 [12]. La plupart des copies de HERV sont défectives, portant des mutations «stop » ou de larges délétions, voire des insertions hétérologues comme dans le gène gag HERV-W sur le chromosome 7q [6]. Des copies qui n'avaient pu être séquencées dans des régions particulières de l'ADN humain, des copies non assemblées par les logiciels de séquençage, mais aussi des copies «non-ubiquitaires» (sans site d'insertion fixe dans l'ADN quand elles sont présentes) ont été identifiées dans des études portant sur la famille HERVK [10,14-16]. De telles études devraient être réalisées afin de compléter les données concernant la famille HERV-W. Par ailleurs, il est intéressant de noter qu'une copie de HERV-W (HERV-W-Env) joue un rôle essentiel dans la physiologie humaine. En effet, une séquence, légèrement différente des autres séquences env de HERV-W, située sur la copie intégrée au chromosome 7q (locus ERVWE1), est hautement exprimée dans le placenta [55] $(\rightarrow)$. Elle permet d'induire la fusion du cytotrophoblaste en syncytiotrophoblaste [17]. Elle

$(\rightarrow)$ Voir la Synthèse de J. Medina et al., page 397 de ce numéro pourrait également avoir des propriétés immunosuppressives qui permettraient la tolérance immunitaire de la mère vis-à-vis du fœtus [18].

\section{Les HERV dans la schizophrénie et le trouble bipolaire}

En 1984, Crow postulait que la schizophrénie (SCZ) pourrait résulter d'une intégration rétrovirale à proximité du gène cérébral de la latéralité [19]. Quelques années plus tard, Deb-Rinker et al. mettaient, pour la première fois, en évidence la présence de la séquence du HERV-W chez trois paires de jumeaux monozygotes discordants pour la SCZ [20]. Depuis, plusieurs études se sont attachées à rechercher des preuves de la réactivation des HERV dans les échantillons de cerveaux de patients (Tableau I). II a ainsi été proposé l'existence d'une surexpression de I'ARN HERV-K pol chez les patients SCZ et TB par rapport aux témoins mais sans 
différence en ce qui concerne les HERV-W [21]. D'autres auteurs ont mis en évidence (1) une augmentation de $45 \%$ de la transcription de HERV-W pol dans le cortex frontal des patients SCZ; (2) une augmentation de l'activité transcriptase inverse dans le cervelet de ces patients; et (3) une augmentation de la transcription de HERVK10 dans le cortex frontal des patients présentant un TB [22]. Une diminution d'expression de HERV-W gag a pu être également mise en évidence dans le cortex cingulaire antérieur ${ }^{1}$ et l'hippocampe ${ }^{2}$ des patients SCZ mais uniquement dans l'hippocampe chez les patients présentant un TB [23]. Ces résultats peuvent paraître discordants. Cependant, il existe de nombreuses limitations à ces études: (1) elles ne se sont en effet pas toutes focalisées sur la recherche des mêmes familles d'HERV ; (2) elles n'utilisent pas les mêmes techniques d'analyses ; et (3) elles ne prennent pas en compte les variables confondantes comme l'utilisation de drogue, d'alcool ou d'antipsychotiques de seconde génération qui peuvent modifier la transcription des HERV [24]. Malgré la limite de sensibilité des techniques utilisées, probablement pas optimales, HERV-W a été détecté dans le liquide céphalo-rachidien de $29 \%$ des patients entrant dans la schizophrénie durant la phase aiguë, les témoins sains ne présentant aucune copie du rétrovirus [25]. Plusieurs études ont recherché la présence des HERV dans le sérum des patients (Tableau II) et, comparés aux témoins, une augmentation de l'expression de HERV-W gag a été retrouvée dans le sérum de patients entrant dans la schizophrénie [26]. Cette augmentation a également été retrouvée au niveau des cellules mononucléées du sang (PBMC) isolées des patients entrant dans la schizophrénie avec une expression accrue de HERV-W gag, de 2 fois par rapport aux témoins [27]. Une corrélation inverse entre niveau d'expression du HERV-W et durée de la maladie semble aussi exister [27] et HERV-W env est surexprimée chez les sujets SCZ et TB [28], associée à une activité transcriptase inverse augmentée d'environ $36 \%$ [29]. La présence dans le sang, d'antigènes codés par HERV-W gag et HERV-W env est observée chez environ la moitié des patients SCZ (des mêmes patients étant positifs pour les deux antigènes, la révélation de HERV-W env étant réalisée par deux anticorps monoclonaux différents) [30]. D’autres rétrovirus ont été isolés comme des séquences proches de ERV-9 pol qui partagent une importante homologie avec HERV-W, les deux étant parfois co-exprimés [31]. Des anticorps présentant des réactions croisées vis-à-vis d'antigènes de rétrovirus animaux ont également pu être isolés chez des patients entrant dans la schizophrénie, mais pas chez des patients ayant présentés plusieurs épisodes de SCZ [32]. Le fait que la durée de la maladie soit une variable confondante importante, ainsi que l'absence de renseignement concernant les traitements des patients inclus, pourraient expliquer ces résultats qui montrent une détection dans seulement une partie des cas étudiés. Alternativement, il pourrait exister des facteurs étiopathogéniques différents chez ces patients dont le diagnostic psychiatrique reste exclusivement clinique.

\footnotetext{
${ }^{1}$ Le cortex cingulaire antérieur est une partie du cortex impliquée dans les processus cognitifs, affectifs mais aussi dans la douleur.

${ }^{2}$ L'hippocampe est situé dans le système limbique et est notamment impliqué dans les processus de
} mémoire.

\section{Physiopathologie des HERV dans la schizophrénie et le trouble bipolaire}

La physiopathologie résultant de la réactivation des HERV dans l'émergence de la SCZ et du TB est complexe et peut être abordée selon différents axes d'interaction. Premièrement, de par leur capacité à s'intégrer aléatoirement au sein du génome, les HERV peuvent modifier l'expression de différents gènes. Par exemple, une insertion d'une copie du HERV-K a été retrouvée dans le gène $P R O D H$ [33]. Ce gène code la proline déshydrogénase, une enzyme essentielle dans la synthèse des différents neuromédiateurs, impliquée dans la SCZ. Un LTR de HERV-W a été identifié dans les régions régulatrices du gène GABBRI [34] qui code le récepteur $G A B A$ de type $B l$ fortement impliqué dans le développement de la SCZ et du TB [35]. Deuxièmement, les HERV peuvent agir au niveau de la cellule. $\varepsilon n$ effet, la protéine d'enveloppe du HERV-W augmente l'expression du BDNF (brain-derived neurotrophic factor), du récepteur neutrophique tyrosine kinase de type 2, du récepteur dopaminergique de type D3 (DRD3), ainsi que la phosphorylation de CREB (C-AMP response element-binding protein) dans les cellules humaines gliales (U251) [29]. Ces modifications ont également été largement associées à la SCZ et aux TB $[36,37]$. Un troisième niveau d'interaction, immunoinflammatoire, est lié à la capacité de HERV-W env d'entraîner un effet pro-inflammatoire en activant l'immunité innée [38] par sa liaison au Toll-like receptor 4 (TLR4) initiant ainsi l'activation de la voie $\mathrm{NF}-\kappa \mathrm{B}$ (nuclear factor-kappa B). Cette stimulation va conduire à la production de cytokines pro-inflammatoires telles que le TNF $\alpha$ (tumor necrosis factor alpha), I'IL(interleukine) l, I'IL6 et de monoxyde d'azote (NO) [38]. Une élévation de ces mêmes cytokines a été observée chez les patients TB et SCZ. En effet, des taux élevés de TNF $\alpha$, ILl et IL6 sont associés aux phases maniaques et dépressives des sujets TB. De plus, les TB sont associés à une augmentation des niveaux d'expression de la NO synthase (NOS). HERV-W env, en activant le TLR4 présent sur les cellules précurseurs d'oligodendrocytes, va induire une inhibition de leur différenciation et de leur capacité de synthétiser les protéines de la myéline, à l'origine d'un effet cytotoxique impactant directement les processus de réparation et de neuroprotection des structures neuronales [39]. Cette inhibition pourrait également être reliée aux problèmes de connectivités structurales et fonctionnelles secondaires à des anomalies de la substance blanche retrouvées à la fois dans le TB et la SCZ [40]. 


\begin{tabular}{|c|c|c|c|c|}
\hline Publications & $\begin{array}{l}\text { Témoins } \\
\text { (nombre de sujets) }\end{array}$ & $\begin{array}{l}\text { Schizophrénie } \\
\text { (nombre de patients) }\end{array}$ & $\begin{array}{l}\text { Troubles bipolaires } \\
\text { (nombre } \\
\text { de patients) }\end{array}$ & Méthode \\
\hline $\begin{array}{l}\text { Karlsson et al. (2004) } \\
\text { [29] }\end{array}$ & $\begin{array}{l}\text { Augmentation de HERV-W } \\
\text { gag chez } 2 \text { témoins } \\
\text { (46) }\end{array}$ & $\begin{array}{l}\text { Augmentation de HERV-W gag dans } \\
9 \text { cas ( } 54 \text { ) }\end{array}$ & & $\begin{array}{l}\text { - Plasma } \\
\text { - RT-PCR }\end{array}$ \\
\hline Yao et al. (2008) [30] & $\begin{array}{l}\text { Expression basale } \\
\text { relative de HERV-W } \\
\text { (26) }\end{array}$ & $\begin{array}{l}\text { - Augmentation par 2,1 de l'expres- } \\
\text { sion relative de HERV-W, comparé } \\
\text { aux témoins ( } 30 \text { ) } \\
\text { - Pas de différence dans l'expres- } \\
\text { sion de HERV-W env }\end{array}$ & & $\begin{array}{l}\text { - PBMC (sang) } \\
\text { - RT-PCR }\end{array}$ \\
\hline Huang et al. (2006) [35] & $\begin{array}{l}\text { Pas de différence dans } \\
\text { l'expression de ERV-9 } \\
\text { pol (38) }\end{array}$ & $\begin{array}{l}\text { Augmentation de } \varepsilon R V-9 \text { pol chez } 20 \\
\text { patients (58) }\end{array}$ & & $\begin{array}{l}\text { - Sang total } \\
\text { - RT-PCR }\end{array}$ \\
\hline Perron et al. (2008) [33] & $\begin{array}{l}\text { - Antigénémie positive } \\
\text { pour HERV-W env chez } 1 \\
\text { témoin (30) } \\
\text { - Antigénémie positive } \\
\text { pour HERV-W gag chez } 2 \\
\text { témoins (49) }\end{array}$ & $\begin{array}{l}\text { - Antigénémie positive pour HERV-W } \\
\text { env chez } 23 \text { patients ( } 49 \text { ) } \\
\text { - Antigénémie positive pour HERV-W } \\
\text { gag chez } 24 \text { patients ( } 49 \text { ) }\end{array}$ & & $\begin{array}{l}\text { - Sérum } \\
\text { - ELISA }\end{array}$ \\
\hline Huang et al. (2010) [32] & $\begin{array}{l}\text { Pas de différence dans } \\
\text { l'expression de HERV-W } \\
\text { env (106) }\end{array}$ & $\begin{array}{l}\text { - Augmentation de l'expression } \\
\text { de HERV-W env chez } 42 \text { patients } \\
\text { entrant dans la schizophrénie (EDS) } \\
\text { (118) } \\
\text { - Augmentation de l'activité RT } \\
\text { de } 35,6 \%\end{array}$ & & $\begin{array}{l}\text { - Plasma } \\
\text { - RT-PCR }\end{array}$ \\
\hline $\begin{array}{l}\text { Dickerson et al. (2012) } \\
\text { [36] }\end{array}$ & Pas d'AC (235) & $\begin{array}{l}\text { - Ac anti-rétrovirus endogènes ani- } \\
\text { maux chez les patients entrant dans } \\
\text { la schizophrénie (EDS) (163) } \\
\text { - Pas d’Ac chez les schizophrènes } \\
\text { avec plusieurs épisodes (268) }\end{array}$ & & $\begin{array}{l}\text { - Sang total } \\
\text { - ELISA }\end{array}$ \\
\hline Perron et al. (2012) [31] & $\begin{array}{l}\text { Expression basale } \\
\text { (73) }\end{array}$ & $\begin{array}{l}\text { Augmentation significative de } \\
\text { HERV-W env (45) }\end{array}$ & $\begin{array}{l}\text { Augmentation de HERV-W } \\
\text { env (45) }\end{array}$ & $\begin{array}{l}\text { - PBMC (sang) } \\
\text { - RT-PCR }\end{array}$ \\
\hline
\end{tabular}

Tableau II. Présence des HERV dans le sérum des patients schizophrènes et bipolaires. HERV-W : human endogenous retrovirus type W; RT-PCR : reverse transcriptase-polymerase chain reaction; PBMC : peripheral blood mononuclear cell; Ac : anticorps; RT : reverse transcriptase.

\section{La séquence d'activation des HERV dans les troubles psychotiques : hypothèse}

Bien que la séquence exacte menant au développement de la SCZ et du TB soit toujours inconnue, un modèle à «deux temps » a été proposé [41]. Le «premier temps» prendrait place durant la grossesse. De nombreux événements survenant pendant cette période comme des infections virales par le cytomégalovirus (CMV), par exemple, ou par des parasites (comme Toxoplasma gondii) ont été associés au développement ultérieur de ces maladies psychiatriques, en particulier dans les troubles bipolaires et la schizophrénie [42, 43]. Ces mêmes pathogènes ont également le potentiel de réactiver in vitro des éléments de la famille HERV-W [44]. Une infection durant la grossesse pourrait donc réactiver des copies de HERV-W entraînant ainsi leur rétrotransposition et/ou des remaniements nucléotidiques dans différents gènes cibles et en particulier ceux impliqués dans le neurodéveloppement. Ces modifications somatiques de I'ADN apparaissant in utero seraient à l'origine d'une prédisposition neurodéveloppementale chez l'enfant à naître [45-48]. Le « second temps» surviendrait plus tard dans la vie du sujet, à la faveur 
d'une nouvelle infection par ces mêmes agents. Celle-ci, ou celles-ci, réactiverai(en)t les $H \varepsilon R V$, avec pour conséquence la synthèse de la protéine d'enveloppe HERV-W qui, par son interaction avec le TLR4, activerait une cascade immunitaire pro-inflammatoire à l'origine d'un état de neuro-inflammation et de neurotoxicité que l'on retrouve dans certains cas de SCZ et de TB, menant alors à l'éclosion et/ou à l'évolution de la maladie.

\section{Conclusions}

Une expression anormale des HERV-W semble donc impliquée dans environ $50 \%$ des SCZ ainsi que dans les TB. L'approche innovante de la physiopathologie des psychoses que nous décrivons, pourrait conduire au développement de nouvelles stratégies thérapeutiques, ciblant plus spécifiquement les mécanismes étiologiques sous-jacents et permettant le traitement de sous-groupes de patients identifiés par des taux élevés d'antigènes de HERV. En plus de la SCZ et des TB, le HERV-W semble également impliqué dans le développement de la sclérose en plaque. Une prise en charge thérapeutique de cette pathologie repose sur l'utilisation d'interféron $\beta$ (IFN- $\beta$ ), qui a montré une efficacité sur l'inhibition de l'expression du rétrovirus [49]. Dans l'optique de l'hypothèse de l'origine rétrovirale de la SCZ et du TB, il est intéressant de noter qu'un article récent rapporte l'efficacité de I'IFN- $\beta$ dans la prise en charge psychopharmacologique des SCZ résistantes [50]. Récemment, le GNbACl, un anticorps dirigé contre la protéine d'enveloppe du HERV-W, a prouvé son efficacité en diminuant la transcription du rétrovirus mais aussi en prévenant son effet sur l'activation pro-inflammatoire des cellules immunitaires et sur la différenciation des oligodendrocytes [51-53]. En se fondant sur l'implication du HERV-W dans la SCZ et le TB, il semble donc possible d'espérer une stratégie thérapeutique dans ces pathologies neuropsychiatriques similaire à celle appliquée dans les maladies neurodégénératives comme la sclérose en plaque $[54,55](\rightarrow)$. $\rightarrow$ Voir la Synthèse de J. Medina et al., page 397 de ce numéro
D'autres études prenant en compte les nombreux facteurs de confusions évoqués et comprenant des cohortes de patients plus importantes sont nécessaires afin de comprendre le rôle que pourrait jouer HERV-W dans l'émergence de la SCZ et du TB. Une meilleure caractérisation des patients, fondée sur des biomarqueurs spécifiques, présentant une signature biologique témoin de la réactivation de HعRV, permettrait d'identifier des sous-groupes spécifiques. Ceci pourrait permettre une prise en charge thérapeutique plus ciblée sur les mécanismes physiopathologiques sous-jacents, ouvrant ainsi la voie à la psychiatrie personnalisée. $\diamond$

\section{SUMMARY}

Implication of human endogenous retroviruses in schizophrenia and bipolar disorder

Schizophrenia and bipolar disorder are neuropsychiatric disorders of unknown origin. It seems that these two disorders share some common etiopathogenic mechanisms including genetic, environmental and inflammatory ones. Reactivation of the human endogenous retrovirus type $W(H \varepsilon R V-W)$ can be a shared element in the pathophysiology of schizophrenia and bipolar disorder, linked to immunogenetic and environment risk factors. We will present studies that have highlighted the presence of HERV-W in schizophrenic and bipolar disorder patients. We will then describe a two-hit model which could explain the common pathophysiological mechanism of affective and non-affective psychosis. Identification of immunoinflammatory mediated subgroup of schizophrenia and bipolar disorder associated to HERV-W reactivation might open the way for the development of diagnostic biomarker and more targeted treatments. These new tools pave the way towards personalized psychiatry for a better care of patients. $\diamond$

\section{LIENS D'INTÉRÊT}

Les auteurs déclarent n'avoir aucun lien d'intérêt concernant les données publiées dans cet article.

\section{RÉFÉRENCES}

1. Lichtenstein P, Yip BH, Björk C, et al. Common genetic determinants of schizophrenia and bipolar disorder in Swedish families: a population-based study. Lancet $2009 ; 373: 234-9$.

2. Benros ME, Eaton WW, Mortensen PB. The epidemiologic evidence linking autoimmune diseases and psychosis. Biol Psychiatry $2014 ; 75$ : 300-6.

3. Kayser MS, Dalmau J. Anti-NMDA receptor encephalitis, autoimmunity, and psychosis. Schizophr Res $2016 ; 176: 36-40$.

4. Lander $E S$, Linton LM, Birren $B$, et al. Initial sequencing and analysis of the human genome. Nature $2001 ; 409: 860-921$.

5. Bannert N, Kurth R. The evolutionary dynamics of human endogenous retroviral families. Annu Rev Genom Hum Genet 2006 ; 7 : 149-73.

6. Weiss RA. The discovery of endogenous retroviruses. Retrovirology 2006 ; $3: 67$.

7. Belshaw R, Katzourakis A, Paces J, et al. High copy number in human endogenous retrovirus families is associated with copying mechanisms in addition to reinfection. Mol Biol Evol $2005 ; 22: 814-7$.

8. Belshaw R, Pereira V, Katzourakis A, et al. Long-term reinfection of the human genome by endogenous retroviruses. Proc Natl Acad Sci USA 2004 ; $101: 4894-9$.

9. Parseval $\mathrm{N}$ de, Heidmann T. Human endogenous retroviruses: from infectious elements to human genes. Cytogenet Genome Res $2005 ; 110$ : 318-32.

10. Marchi $\varepsilon$, Kanapin A, Magiorkinis G, et al. Unfixed endogenous retroviral insertions in the human population. J Virol $2014 ; 88$ : 9529-37.

11. Buzdin A. Human-specific endogenous retroviruses. ScientificWorldJournal $2007 ; 7:$ : 1848-68.

12. Kim HS. Genomic impact, chromosomal distribution and transcriptional regulation of HERV elements. Mol Cells 2012 ; 33 : 539-44.

13. Voisset C, Bouton O, Bedin F, et al. Chromosomal distribution and coding capacity of the human endogenous retrovirus HERV-W family. AIDS Res Hum Retrovir $2000 ; 16: 731-40$.

14. Wildschutte JH, Williams ZH, Montesion M, et al. Discovery of unfixed endogenous retrovirus insertions in diverse human populations. Proc Natl Acad Sci USA 2016 ; 113 : ع2326-34.

15. Wildschutte JH, Ram D, Subramanian R, et al. The distribution of insertionally polymorphic endogenous retroviruses in breast cancer patients and cancer-free controls. Retrovirology $2014 ; 11: 62$.

16. Marchi $\varepsilon$, Kanapin $A$, Byott $M$, et al. Neanderthal and Denisovan retroviruses in modern humans. Curr Biol CB $2013 ; 23:$ R994-5.

17. Blond JL, Besème F, Duret L, et al. Molecular characterization and placental expression of HERV-W, a new human endogenous retrovirus family. J Virol $1999 ; 73: 1175-85$.

18. Wenner M. Virology: the battle within. Nature $2008 ; 451$ : 388-9.

19. Crow TJ. Is schizophrenia an infectious disease? Lancet 1983 ; $1: 173-5$.

20. Deb-Rinker P, Klempan TA, O'Reilly RL, et al. Molecular characterization of a MSRV-like sequence identified by RDA from monozygotic twin pairs discordant for schizophrenia. Genomics $1999 ; 61: 133-44$. 


\section{RÉFÉRENCES}

21. Frank 0 , Giehl M, Zheng $C$, et al. Human endogenous retrovirus expression profiles in samples from brains of patients with schizophrenia and bipolar disorders. J Virol $2005 ; 79: 10890-901$.

22. Yolken RH, Karlsson H, Yee F, et al. Endogenous retroviruses and schizophrenia. Brain Res Brain Res Rev 2000 ; 31 : 193-9.

23. Weis $S$, Llenos IC, Sabunciyan $S$, et al. Reduced expression of human endogenous retrovirus (HERV)-W GAG protein in the cingulate gyrus and hippocampus in schizophrenia, bipolar disorder, and depression. J Neural Transm Vienna Austria 19962007 ; 114 : 645-55.

24. Diem 0 , Schäffner $M$, Seifarth $W$, et al. Influence of antipsychotic drugs on human endogenous retrovirus (HERV) transcription in brain cells. PloS One $2012 ; 7$ : e30054.

25. Karlsson H, Bachmann S, Schröder J, et al. Retroviral RNA identified in the cerebrospinal fluids and brains of individuals with schizophrenia. Proc Natl Acad Sci USA $2001 ; 98$ : 4634-9.

26. Karlsson H, Schröder J, Bachmann S, et al. HERV-W-related RNA detected in plasma from individuals with recent-onset schizophrenia or schizoaffective disorder. Mol Psychiatry $2004 ; 9$ : $12-3$.

27. Yao $Y$, Schröder J, Nellåker $C$, et al. Elevated levels of human endogenous retrovirus-W transcripts in blood cells from patients with first episode schizophrenia. Genes Brain Behav 2008 ; $7: 103-12$

28. Perron H, Hamdani N, Faucard R, et al. Molecular characteristics of human endogenous retrovirus type-W in schizophrenia and bipolar disorder. Transl Psychiatry $2012 ; 2$ : e201.

29. Huang W, Li S, Hu Y, et al. Implication of the env gene of the human endogenous retrovirus $W$ family in the expression of BDNF and DRD3 and development of recent-onset schizophrenia. Schizophr Bull $2011 ; 37$ : 988-1000.

30. Perron H, Mekaoui L, Bernard C, et al. Endogenous retrovirus type W GAG and envelope protein antigenemia in serum of schizophrenic patients. Biol Psychiatry $2008 ; 64: 1019-23$.

31. Perron H, Garson JA, Bedin F, et al. Molecular identification of a novel retrovirus repeatedly isolated from patients with multiple sclerosis. The Collaborative research group on multiple sclerosis. Proc Natl Acad Sci USA 1997 ; 94 : 7583-8.

32. Dickerson F, Lillehoj $\varepsilon$, Stallings $C$, et al. Antibodies to retroviruses in recent onset psychosis and multi-episode schizophrenia. Schizophr Res 2012 ; 138 : 198-205.

33. Suntsova M, Gogvadze $\varepsilon V$, Salozhin $S$, et al. Human-specific endogenous retroviral insert serves as an enhancer for the schizophrenia-linked gene PRODH. Proc Natl Acad Sci USA 2013 ; 110 : 19472 -

34. Hegyi H. GABBRI has a HERV-W LTR in its regulatory region - a possible implication for schizophrenia. Biol Direct $2013 ; 8: 5$.

35. Fatemi SH, Folsom TD, Thuras PD. Deficits in GABA(B) receptor system in schizophrenia and mood disorders: a postmortem study. Schizophr Res 2011 ; $128: 37-43$.

36. Mitchelmore C, Gede L. Brain Derived neurotrophic factor: epigenetic regulation in psychiatric disorders. Brain Res $2014 ; 1586$ : 162-72.

37. Chang $Y H$, Lee SY, Chen SL, et al. Genetic variants of the BDNF and DRD3 genes in bipolar disorder comorbid with anxiety disorder. J Affect Disord 2013 ; $151: 967-72$.

38. Young GR, Stoye JP, Kassiotis G. Are human endogenous retroviruses pathogenic? An approach to testing the hypothesis. BioEssays News Rev Mol Cell Dev Biol 2013 ; 35 : 794-803.

39. Kremer D, Schichel T, Förster M, et al. Human endogenous retrovirus type $W$ envelope protein inhibits oligodendroglial precursor cell differentiation. Ann Neurol 2013 ; 74 : 721-32.

40. Najjar S, Pearlman DM. Neuroinflammation and white matter pathology in schizophrenia: systematic review. Schizophr Res $2015 ; 161: 102-12$.

41. Leboyer M, Tamouza R, Charron D, et al. Human endogenous retrovirus type W (HERV-W) in schizophrenia: a new avenue of research at the gene-environment interface. World J Biol Psychiatry 2013; $14: 80-90$
42. Blomström A, Karlsson $\mathrm{H}$, Wicks $\mathrm{S}$, et al. Maternal antibodies to infectious agents and risk for non-affective psychoses in the offspring: a matched case-control study. Schizophr Res $2012 ; 140: 25-30$.

43. Sutterland AL, Fond G, Kuin A, et al. Beyond the association. Toxoplasma gondii in schizophrenia, bipolar disorder, and addiction: systematic review and meta-analysis. Acta Psychiatr Scand 2015 ; 132 : 161-79.

44. Frank 0 , Jones-Brando L, Leib-Mosch C, et al. Altered transcriptional activity of human endogenous retroviruses in neuroepithelial cells after infection with Toxoplasma gondii.) Infect Dis 2006; 194 : 1447-9.

45. Grozeva D, Kirov G, Ivanov D, et al. Rare copy number variants: a point of rarity in genetic risk for bipolar disorder and schizophrenia. Arch Gen Psychiatry $2010 ; 67: 318-27$

46. Stefansson H, Rujescu D, Cichon S, et al. Large recurrent microdeletions associated with schizophrenia. Nature $2008 ; 455$ : 232-6.

47. International schizophrenia consortium. Rare chromosomal deletions and duplications increase risk of schizophrenia. Nature 2008 ; 455 : 237-41.

48. Bassett AS, Marshall CR, Lionel AC, et al. Copy number variations and risk for schizophrenia in 22q11.2 deletion syndrome. Hum Mol Genet 2008; 17 : 4045-53.

49. Petersen T, Møller-Larsen A, Ellermann-Eriksen S, et al. Effects of interferonbeta therapy on elements in the antiviral immune response towards the human herpesviruses EBV, HSV, and VZV, and to the human endogenous retroviruses HERV-H and HERV-W in multiple sclerosis. J Neuroimmunol 2012 ; 249 : 105-8

50. Grüber L, Bunse T, Weidinger $\varepsilon$, et al. Adjunctive recombinant human interferon gamma-lb for treatment-resistant schizophrenia in 2 patients. $J$ Clin Psychiatry $2014 ; 75: 1266-7$.

51. Derfuss T, Curtin F, Guebelin C, et al. A phase lla randomized clinical study testing $\mathrm{GNbACl}$, a humanized monoclonal antibody against the envelope protein of multiple sclerosis associated endogenous retrovirus in multiple sclerosis patients: a twelve month follow-up. J Neuroimmunol 2015 ; 285 : 68-70.

52. Curtin F, Perron H, Kromminga A, et al. Preclinical and early clinical development of $\mathrm{GNbACl}$, a humanized IgG4 monoclonal antibody targeting endogenous retroviral MSRV-Env protein. mAbs $2015 ; 7: 265-75$.

53. Kremer $D$, Förster $M$, Schichel $T$, et al. The neutralizing antibody GNbAC abrogates HERV-W envelope protein-mediated oligodendroglial maturation blockade. Mult Scler 2015 ; 21 : 1200-3.

54. Medina J, Perron H. Séquences provenant d'éléments génétiques mobiles, face cachée du génome humain. Med Sci (Paris) 2017 ; 33 : 151-8.

55. Medina J, Charvet $B$, Horvat $B$, et al. Des séquences rétrovirales endogènes dans le génome humain peuvent jouer un rôle physiologique ou pathologique. Med Sci (Paris) 2017 ; $33: 397-403$.

\section{LA FONDATION PREMUP : UN OPÉRATEUR DE TERRAIN EN PÉRINATALITÉ RECONNU POUR SON EXCELLENCE ET SON INTERDISCIPLINARITÉ}

La Fondation de coopération scientifique PremUp, unique en Europe, intervient sur la prévention du handicap à la naissance, par la protection de la santé de la femme enceinte et du nouveau-né.

TIRÉS À PART

P. Ellul

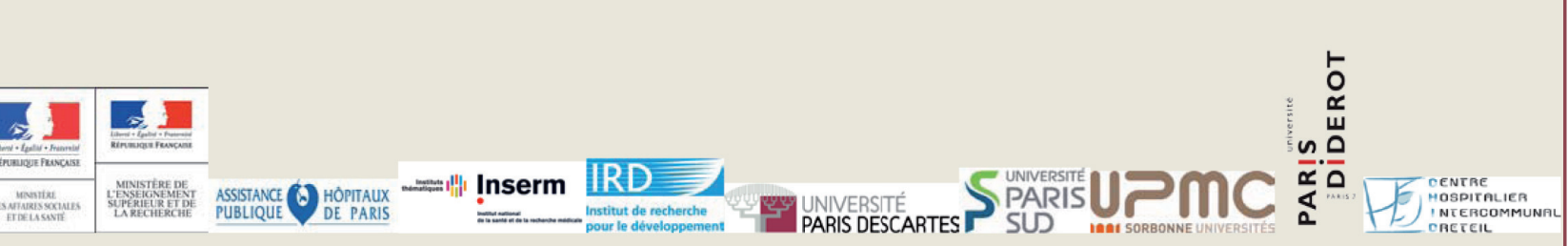

